\title{
Worst-case analysis of electronic circuits based on an analytic forward solver approach
}

\author{
Mario Schenk, Annette Muetze and Klaus Krischan \\ Electric Drives and Machines Institute (EAM), \\ Graz University of Technology, Graz, Austria, and \\ Christian Magele \\ Institute of Fundamentals and Theory in Electrical Engineering (IGTE), \\ Graz University of Technology, Graz, Austria
}

Worst-case analysis of electronic circuits

Received 14 December 2018 Revised 28 May 2019 Accepted 29 May 2019

\begin{abstract}
Purpose - The purpose of this paper is to evaluate the worst-case behavior of a given electronic circuit by varying the values of the components in a meaningful way in order not to exceed pre-defined currents or voltages limits during a transient operation.

Design/methodology/approach - An analytic formulation is used to identify the time-dependent solution of voltages or currents using proper state equations in closed form. Circuits with linear elements can be described by a system of differential equations, while circuits composing nonlinear elements are described by piecewise-linear models. A sequential quadratic program (SQP) is used to find the worst-case scenario.
\end{abstract}

Findings - It is found that the worst-case scenario can be obtained with as few solutions to the forward problem as possible by applying an SQP method.

Originality/value - The SQP method in combination with the analytic forward solver approach shows that the worst-case limit converges in a few steps even if the worst-case limit is not on the boundary of the parameters.

Keywords Computer-aided design, Transient analysis, Circuit analysis, Power electronic simulation, Circuit simulation, SQP, Time-domain analysis

Paper type Research paper

\section{Introduction}

Electronic components always come along with certain tolerances; therefore, worst-case dimensioning of electronic circuits composed of such components has been gaining more and more importance. Some simulators [e.g. LTspice (Analog Devices, 2019) and PSpice (Orcad PSpice, 2019)] offer the possibility for a Monte Carlo (MC) analysis. This analysis provides statistical data on the impact of a device parameter's variance. A major disadvantage of stochastic methods is that they require a high number of simulation runs to reach the worst-case limits. This can be improved advantageously by solving currents

(C) Mario Schenk, Annette Muetze, Klaus Krischan and Christian Magele. Published by Emerald Publishing Limited. This article is published under the Creative Commons Attribution (CC BY 4.0) licence. Anyone may reproduce, distribute, translate and create derivative works of this article (for both commercial and non-commercial purposes), subject to full attribution to the original publication and authors. The full terms of this licence may be seen at http://creativecommons.org/licences/by/4.0/legalcode 
COMPEL 38,5

and voltages analytically. The proposed approach allows conducting a full search over the parameter space. This, in turn, provides the possibility of worst-case analyses of the different parameters of interest. (In our example cases the maximum current that may occur was chosen.) Additionally, it allows full flexibility in modeling the individual components and their parameters. Furthermore, simulation results are extremely compact and can theoretically be stored with arbitrary precision. Finally, the objective function $f(\mathbf{x})$ needed for any optimizer is available in analytic form. In this paper, the analytic forward solver approach (AFSA) and the sequential quadratic program (SQP) are implemented in Maple (Maplesoft, 2019), a computer algebra system (CAS). Section 2 explores the analytic forward solver approach and the SQP approach. Section 3 describes the chosen example case application, a closed-loop flyback converter and compares the performance of the SQP method with an MC analysis and evolution strategy. Examples for interesting references in the context of worst-case and sensitivity analyses are given by Chiariello et al. (2015), Khaligh et al. (2006); Lian (2012).

\section{Method overview}

\subsection{Analytic forward solver approach}

Today, many circuit simulators for electronic circuits with different approaches are available. The proposed approach uses analytic solution techniques and has been developed especially for optimization and worst-case dimensioning of small-scale electronic circuits. The advantages are:

- the full flexibility in the modeling of the individual components and their parameters;

- simulation results are extremely compact and can theoretically be stored with arbitrary precision; even with a high number of simulation runs, the generated data remain easy to handle; and

- analytic methods show the potential of more efficient parameter studies.

While a comprehensive review of all existing circuit simulators is not within the scope of this paper, a short overview is nevertheless provided, for the sake of completion. The analytic forward solver approach supports analytic time-domain transient analysis for switched networks with piecewise-linear models and uses analytic methods for solving the systems of ordinary differential equations (ODEs). Symbolic simulators like ISAAC (Gielen et al., 1989), SAPWIN (Liberatore et al., 1995; Fontana et al., 2015) and Analog Insyde (Thomassian, 2007) exist. These simulators do not support fully analytic timedomain transient analysis for switched networks with piecewise-linear models. The simulator for integrated switched-mode power supplies circuits (SISMPSC) (Cliquennois and Trochut, 2007) is based on symbolic calculus tools and supports symbolic state-space equations (SSSE) but uses numerical methods for solving the systems of ordinary differential equations (ODEs). For the elemental circuit description the analytic forward solver approach uses a special Circuit-Model instead of the widely used netlist as, for example, used in the different implementations of the core SPICE algorithm SPICE2 (Nagel, 1975), LTspice and PSpice. A Circuit-Model describes the electronic circuit with symbolic ordinary differential equations, if state variables are present. In the case of no state variables, the electronic circuit is described by symbolic algebraic equations. The electronic circuit to be simulated may contain linear and nonlinear components. Linear parts are described directly with a SubCircuit-Model, an extended symbolic state-space model (ESSSM) and nonlinear ones with a Circuit-Model, comprising several SubCircuit- 
Models itself, the associated boundary conditions and a state table. The fulfilled boundary conditions of the active SubCircuit-Model are the reference (input for the state table) for the next SubCircuit-Models. An example of a SubCircuit-Model is shown in Figure 1.

The associated ESSSM is described by (1)-(3). Equation (1) represents the system of differential (state) equations, (2) the signal of interest, and (3) the I/O interface. In this example, the input-cell $\mathbf{y}_{\mathrm{I}}(\mathrm{t})$ of the I/O interface is not defined:

$$
\begin{gathered}
{\left[\begin{array}{c}
L_{1} \frac{\mathrm{d} I_{\mathrm{L} 1}(t)}{\mathrm{d} t} \\
C_{1} \frac{\mathrm{d} V_{\mathrm{C} 1}(t)}{\mathrm{d} t}
\end{array}\right]=\left[\begin{array}{cc}
-R_{1} & -1 \\
1 & 0
\end{array}\right]\left[\begin{array}{l}
I_{\mathrm{L} 1}(t) \\
V_{\mathrm{C} 1}(t)
\end{array}\right]+\left[\begin{array}{c}
V_{\text {in }} \sin (\omega t) \\
-I_{\text {out }}(t)
\end{array}\right]} \\
\mathbf{y}(t)=I_{\mathrm{L} 1}(t)
\end{gathered}
$$

I/O interface:

$$
\begin{gathered}
\mathbf{y}_{\mathrm{IO}}(t)=\left[\begin{array}{c}
\mathbf{y}_{\mathrm{I}}(t) \\
\mathbf{y}_{\mathrm{O}}(t)
\end{array}\right] \quad \text { with } \\
\mathbf{y}_{\mathrm{I}}(t)=[] \quad \mathbf{y}_{\mathrm{O}}(t)=\left[\begin{array}{l}
I_{\text {out }}(t) \\
V_{\mathrm{C} 1}(t)
\end{array}\right]
\end{gathered}
$$

A collection of predefined SubCircuit-Models is provided for the individual circuit design. Connecting such simple predefined SubCircuit-Models results in a new SubCircuit-Model. This results in a large number of possible SubCircuit-Models. The advantage of this approach is that the electronic circuit to be simulated can be built from such SubCircuitModels without transformation to a state-space model; only the I/O definitions must be substituted.

The ODE system solver module generally solves the ODE system from each SubCircuitModel. The proposed approach uses the built-in ODE solver from Maple. Out of the different solver methods and options available, the proposed approach uses the Laplace method. When the ODE system from each SubCircuit-Model has been solved once, the solution is stored and, therefore, the ODE system does not need to be solved again. The analytic solution of the ODE System (1) for $I_{\mathrm{L} 1}(\mathrm{t})$ from the SubCircuit-Model in Figure 1 is shown (5). The initial conditions $I_{\mathrm{L} 1}(0), V_{\mathrm{C} 1}(0)$ and $I_{\text {out }}(\mathrm{t})$ were set to 0 .

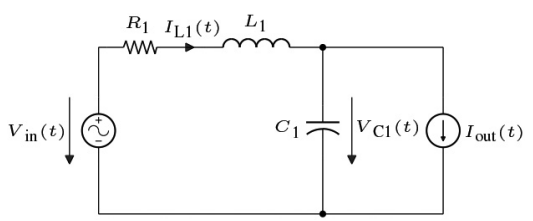

Figure 1. SubCircuit-Model: RLC series resonant circuit 
COMPEL

38,5

1658

$$
\begin{aligned}
I_{\mathrm{L} 1}(t)= & \frac{M_{1}}{4 k L_{1}}\left(E_{1}\left(C_{1} R_{1} k \omega+M_{2}\right)+D_{0}\right) \\
& -\frac{M_{1}}{4 k L_{1}}\left(E_{2}\left(C_{1} R_{1} k \omega-M_{2}\right)\right) \\
k= & \sqrt{\left(C_{1}\left(C_{1} R_{1}^{2}-4 L_{1}\right)\right)} \\
D_{0}= & 4 V_{\text {in }} C_{1} L_{1} k \omega\left(1-C_{1} L_{1} \omega^{2}\right) \cos (\omega t) \\
& +4 V_{\text {in }} C_{1}^{2} L_{1} R_{1} k \omega^{2} \sin (\omega t) \\
E_{1}= & V_{\text {in }}\left(-C_{1} R_{1}+k\right) \mathrm{e}^{\left.\frac{1}{2} C_{1} R_{1} L_{1}\right) t} \\
E_{2}= & V_{\text {in }}\left(C_{1} R_{1}+k\right) \mathrm{e}^{\left.-\frac{1}{2} C_{1} R_{1}+k\right) t} \\
M_{1} L_{1} & \frac{1}{\omega^{4} C_{1}^{2} L_{1}^{2}+\omega^{2} C_{1}^{2} R_{1}^{2}-2 \omega^{2} C_{1} L_{1}+1} \\
M_{2}= & 2 C_{1}^{2} L_{1}^{2} \omega^{3}+C_{1}^{2} R_{1}^{2} \omega-2 C_{1} L_{1} \omega
\end{aligned}
$$

The time-domain transient analysis for a Circuit-Model starts at the first SubCircuit-Model, then, the boundary conditions for this SubCircuit-Model are verified. The fulfilled boundary condition determines the next SubCircuit-Model and the analytic solution for that time interval. This is repeated until the final circuit operating time to be simulated $t_{\text {Sim, }}$, is reached.

\subsection{Sequential quadratic program approach}

The implemented SQP method (6) is based on an active set strategy with linear inequality constraints (7) (Fletcher, 2000):

$$
\begin{gathered}
\min _{\mathbf{x} \in \mathbf{R}^{n}} \quad f(\mathbf{x})=\frac{1}{2} \mathbf{x}^{T} \mathbf{H} \mathbf{x}+\mathbf{c}^{T} \mathbf{x} \\
\text { subject to } \quad \mathbf{A} \mathbf{x} \geq \mathbf{b}
\end{gathered}
$$

where:

$\mathbf{x}=$ column vector of the device parameters;

$f(\mathbf{x})=$ objective function;

$\mathbf{H}=$ Hessian matrix of the objective function;

c = gradient of the objective function;

A $=$ constant $m \times n$ matrix;

$\mathbf{b}=$ constant column vector $\mathbf{b} \in \mathbf{R}^{m}$;

$n$ = number of parameters; and

$m$ = number of constraints.

The Hessian matrix $\mathbf{H}$ is updated in each iteration step until the optimal solution is found. To apply an SQP strategy, the objective function $f(\mathbf{x})$ must be defined; for example, the inductor current (2) shown in Figure 1. The SQP strategy generally finds the minimum of the objective function $f(\mathbf{x})$, in case of the maximum $-f(\mathbf{x})$ must be used instead. The inequality constraints (7) are constructed from the device parameter bounds. In case of $n$ device parameters, it is $m=2 n$. The general form of (7) in matrix form is described in (8). 


$$
\left[\begin{array}{cccc}
1 & 0 & \cdots & 0 \\
-1 & 0 & \cdots & 0 \\
\vdots & & \ddots & \vdots \\
0 & 0 & \cdots & 1 \\
0 & 0 & \cdots & -1
\end{array}\right]\left[\begin{array}{c}
x_{1} \\
x_{1} \\
\vdots \\
x_{n} \\
x_{n}
\end{array}\right] \geq\left[\begin{array}{c}
x_{1 \min } \\
-x_{1 \max } \\
\vdots \\
x_{n \min } \\
-x_{n \max }
\end{array}\right]
$$

\subsection{Worst-case analysis of a resistor inductor capacitor (RLC) series resonant circuit}

The schematic of the RLC series resonant circuit is shown in Figure 1. Table I summarizes the values of the components and the SQP parameters. The maximum peak inductor current $\hat{I}_{\mathrm{L} 1}$ in steady-state should be determined as a function of two parameters, e.g. $t$ and $L_{1}$. The objective function $f(\mathbf{x})$ for the minimum peak inductor current is obtained by the evaluation of the component values from Table I in (5). The objective function for the maximum is:

$$
f(\mathbf{x})=I_{\mathrm{L} 1 \max }\left(t, L_{1}\right)=-I_{\mathrm{L} 1}\left(t, L_{1}\right) .
$$

To find the maximum peak inductor in steady-state, the lower bound for $t$ was chosen to be 10 times larger than the period $T=1 / f$. The 3 -D plot of the objective function $I_{\mathrm{L} 1 \text { max }}\left(t, L_{1}\right)(9)$ including the solution path of the SQP method is illustrated in Figure 2. The implemented SQP method converges to the maximum after 7 iterations (summarized in Table II) with $\left|\hat{I}_{\mathrm{L} 1}\right|=0.04 \mathrm{~A}$ and is also visualized in the contour plot in Figure 3 . The solution of the SQP method is exactly the same as expected: at the resonant frequency, the capacitive and inductive reactances cancel each other and the current through the inductor $L_{1}$ is only limited by the resistor $R_{1}$, hence $\hat{I}_{\mathrm{L} 1}=V_{\text {in }} / R_{1}=0.04 \mathrm{~A}$.

\section{Example case}

The performance of the proposed approach is demonstrated by a worst-case analysis of a flyback converter in continuous conduction mode (CCM). The goal is to determine the maximum magnetizing current $\hat{I}_{\mathrm{LM}}$ from the transformer $T_{1}$ in steady-state. This is especially important for the transformer design. The schematic of the closed-loop flyback converter is shown in Figure 4 and is divided into three parts:

(1) Power stage: includes a real transformer with the winding resistance $R_{\mathrm{P}}$, the magnetizing inductance $L_{\mathrm{M}}$, an ideal transformer $T_{1}$, a power switch $Q_{1}$, a current sense resistor $R_{\text {Sense, }}$ the secondary rectifier $D_{1}$ and the output filter $C_{\mathrm{O}}$. The power

\begin{tabular}{lclc}
\hline $\begin{array}{l}\text { Components } \\
\text { values }\end{array}$ & $\begin{array}{c}\text { Parameter } \\
x_{i}\end{array}$ & Lower bound & Upper bound \\
\hline$V_{\text {in }}=2 \mathrm{~V}$ & $t$ & $t_{\min }=10 \mathrm{~s}$ & $t_{\max }=10.5 \mathrm{~s}$ \\
$R_{1}=50 \Omega$ & $L_{1}$ & $L_{1 \text { min }}=0.1 \mathrm{H}$ & $L_{1 \max }=30 \mathrm{H}$ \\
$C_{1}=15 \mathrm{mF}$ & & \\
$\omega=2 \pi f$ & & & \\
$f=1 \mathrm{~Hz}$ & & & \\
$I_{\text {out }}(t)=0 \mathrm{~A}$ & & & \\
\hline
\end{tabular}

Table I. Component values and SQP parameters RLC series resonant circuit of Figure 1 
COMPEL

38,5

1660

Figure 2.

RLC series resonant

circuit $I_{\mathrm{L} 1 \text { max }}\left(t, L_{1}\right)$

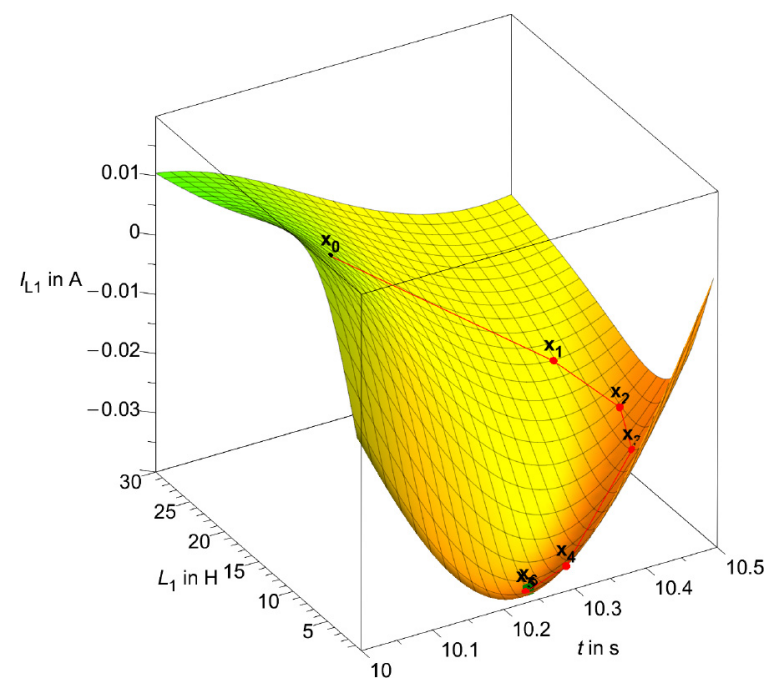

Table II.

SQP Method on RLC series resonant circuit of Figure 1

\begin{tabular}{lccccccc}
\hline$k$ & 0 & 1 & 2 & 3 & 4 & 5 & 6 \\
\hline$t^{(k)}$ in $s$ & 10.1 & 10.41 & 10.46 & 10.44 & 10.29 & 10.24 & 10.25 \\
$L_{1}^{(k)}$ in $\mathrm{H}$ & 15.0 & 15.0 & 9.735 & 5.999 & 0.4609 & 1.452 & 1.692 \\
$I_{\mathrm{L} 1 \max }^{(k)}$ in $\mathrm{mA}$ & 8.062 & -20.53 & -24.58 & -27.31 & -36.25 & -39.98 & -40.0 \\
\hline
\end{tabular}

Figure 3.

RLC series resonant circuit contour plot $I_{\mathrm{L} 1 \max }\left(t, L_{1}\right)$

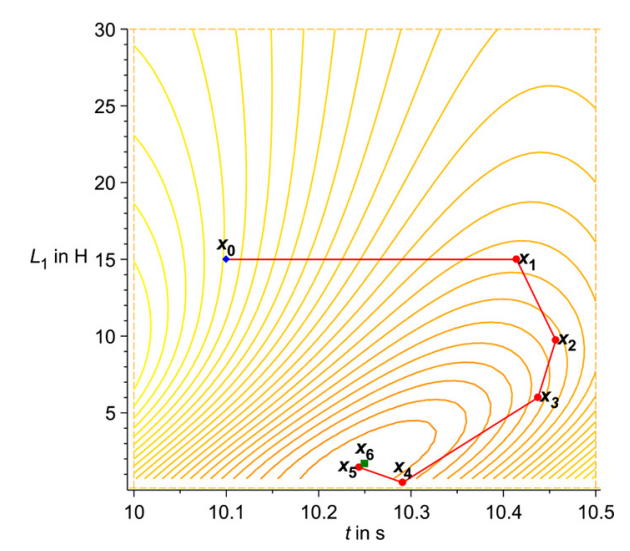

switch $Q_{1}$ is modeled as a voltage controlled ideal switch with two resistors $Q_{1 \mathrm{RDS}(\mathrm{on})}$ representing the resistance in the on-region and the resistance $Q_{1 \mathrm{RDS}(\mathrm{off})}$ for the cut-off region.

(2) PWM controller: for the control method, peak current mode control with constant switching frequency $F_{\mathrm{S}}$ is chosen and is implemented at the PWM controller block. A detailed structure of the PWM controller is shown in Figure 5. 


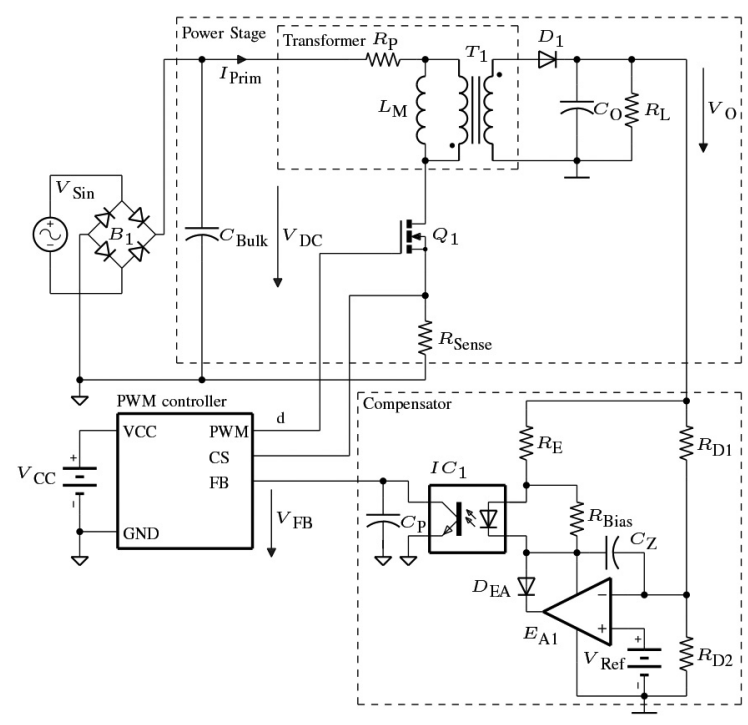

Worst-case analysis of electronic circuits

1661

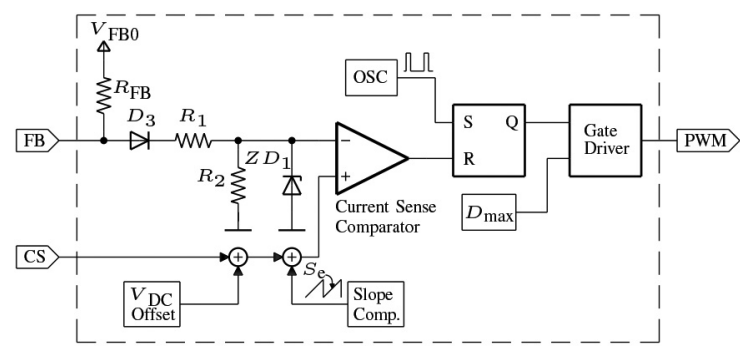

Figure 4. Schematic: a closedloop flyback converter with peak current mode control

Figure 5. Block diagram peak current mode PWM controller

(3) Compensator: a Type II compensator (Ridley, 2011; Basso, 2012) is used, containing the error amplifier $E A_{1}$, a diode $D_{\mathrm{EA}}$, and a voltage reference $V_{\text {Ref }}$ to model an adjustable shunt regulator (such as TL431). The error amplifier $E A_{1}$ is modeled as an ideal amplifier with infinite gain. The diode $D_{\mathrm{EA}}$ is used to add an additional offset to the output level of $E A_{1}$ and also ensures that the amplifier can only sink the current. The optocoupler $I C_{1}$ is modeled in the forward linear region by multiplying $I_{\mathrm{F}}$ with a constant factor, the current transfer ratio (CTR) $I_{\mathrm{C}}=C T R I_{\mathrm{F}}$ and in the saturation region by a constant voltage source $V_{\mathrm{CE}(\mathrm{sat})}$.

In general, many switching cycles are necessary until the system has reached the steady-state behavior in the case of switching mode power supply. In steady-state, a PWM signal with constant duty-cycle $d_{\text {on }}$ is generated in the way that the average output voltage $\overline{V_{\mathrm{O}}}$ equals $V_{\mathrm{O} \text { nom. }}$ The compensator and the PWM controller are responsible for tuning the duty-cycle.

\subsection{Steady-state analysis}

The major advantage of the analytic forward solver approach is that the unknown dutycycle in steady-state can be calculated based on the analytic solutions of the state variables 
COMPEL and/or signal of interest, e.g. (2). In addition, the closed-loop flyback converter can be 38,5 simplified to an open-loop flyback converter or Power Stage. This means that no additional algorithms are needed for the steady-state analysis, e.g. as used in (Li and Tymerski, 2000; Wong et al., 2000; Setiawan et al., 2017; Moskovko and Vityaz, 2018). The flyback converter Power Stage operating in CCM has two SubCircuit-Models (states): Figure 6 shows steadystate waveforms of the state variables for one switching cycle $T_{\mathrm{S}}=1 / F_{\mathrm{S}}$.

- State 1: PWM high, MOSFET $Q_{1}$ is switched on (saturation) and $D_{1}$ switched off (reverse bias) with $0<t \leq t_{\text {on }}$.

- State 2: PWM low, MOSFET $Q_{1}$ is switched off (cut-off) and $D_{1}$ switched on (forward bias) with $t_{\mathrm{on}}<t<T_{\mathrm{S}}$.

The initial conditions of the state variables in state 1 can be expressed as:

$$
\begin{gathered}
I_{\mathrm{LM} 10}=I_{\mathrm{LM} \mathrm{off}}\left(T_{\mathrm{S}}-t_{\mathrm{on}}\right) \\
V_{\mathrm{CO} 10}=V_{\mathrm{CO} \mathrm{off}}\left(T_{\mathrm{S}}-t_{\mathrm{on}}\right)
\end{gathered}
$$

and for state 2 as:

$$
\begin{gathered}
I_{\mathrm{LM} 20}=I_{\mathrm{LM} \mathrm{on}}\left(t_{\mathrm{on}}\right) \\
V_{\mathrm{CO} 20}=V_{\mathrm{CO} \text { on }}\left(t_{\mathrm{on}}\right) . \\
V_{\mathrm{Onom}}=\frac{1}{T_{\mathrm{S}}}\left(\int_{0}^{t_{\mathrm{on}}} V_{\mathrm{CO} 1}(t) \mathrm{d} t+\int_{t_{\mathrm{on}}}^{T_{\mathrm{S}}} V_{\mathrm{CO} 2}(t) \mathrm{d} t\right)
\end{gathered}
$$

Equation (14) expresses that the average voltage $\overline{V_{\mathrm{CO}}}$ on the capacitor $C_{\mathrm{O}}$ equals $V_{\mathrm{O} \text { nom. }}$ Using (10) and (11) in (12) and (13) with (14) results in a system of equations with three unknowns $I_{\mathrm{LM} 20}, V_{\mathrm{CO} 20}$, and $t_{\mathrm{on}}$. For a better performance the system of equations in analytic form (generated by the analytic forward solver approach) are solved numerically.

Figure 6.

Waveforms flyback

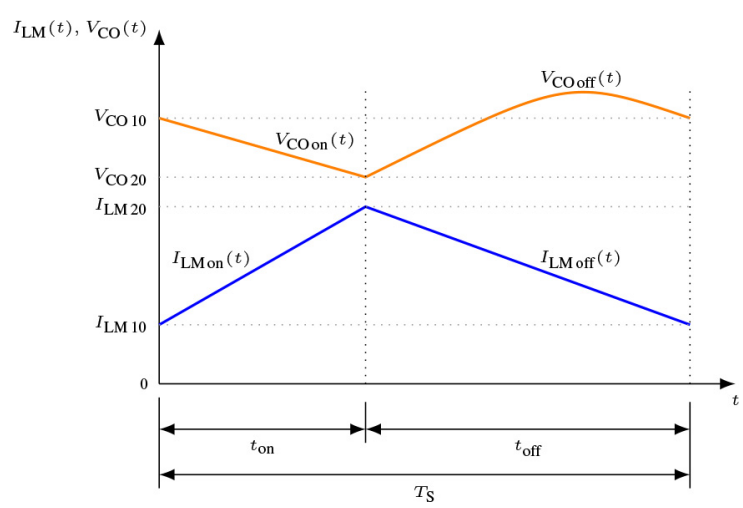




\subsection{Sequential quadratic program result}

The solution for the initial condition $I_{\mathrm{LM}} 20$ of the system of equations (12)-(14) corresponds to $\hat{I}_{\mathrm{LM}}$. The objective function for the maximum magnetizing current is

$$
f(\mathbf{x})=-I_{\text {LM20 }}
$$

Table III summarizes component values and parameters for the closed-loop flyback converter and Table IV summarizes the values the SQP parameters.

The implemented SQP method converges to the maximum after 6 iterations with $\left|\hat{I}_{\mathrm{LM}}\right|=2.583 \mathrm{~A}$.

\subsection{Transient simulation}

To verify the results from the SQP method in steady-state, for each iteration $k$ (summarized in Table V) a transient simulation of the closed-loop flyback converter has been performed with the same parameters in Table IV and III as shown in Figure 7. The transient simulation

\begin{tabular}{lll}
\hline Power stage & PWM controller & Compensator \\
\hline$R_{\mathrm{P}}=0.2 \Omega$ & $V_{\text {PWMlow }}=0 \mathrm{~V}$ & $V_{\text {Ref }}=2.5 \mathrm{~V}$ \\
$T_{1 \text { Ratio }}=0.25$ & $V_{\text {PWM high }}=10 \mathrm{~V}$ & $R_{\mathrm{D} 1}=110 \mathrm{k} \Omega$ \\
$Q_{1 \text { Vth(on) }}=3.5 \mathrm{~V}$ & $S_{\mathrm{e}}=0.01 \frac{\mathrm{V}}{\mu \mathrm{s}}$ & $R_{\mathrm{D} 2}=10 \mathrm{k} \Omega$ \\
$Q_{1 \mathrm{RDS}(\text { on })}=0.34 \Omega$ & $V_{\mathrm{DC} \text { Offset }}=0 \mathrm{~V}$ & $R_{\mathrm{E}}=1 \mathrm{k} \Omega$ \\
$Q_{1 \mathrm{RDS}(\text { off })}=0.1 \mathrm{G} \Omega$ & $V_{\mathrm{FB} 0}=6.6 \mathrm{~V}$ & $R_{\mathrm{Bias}}=1 \mathrm{k} \Omega$ \\
$D_{1 \mathrm{RD}}=50 \mathrm{~m} \Omega$ & $R_{\mathrm{FB}}=1 \mathrm{k} \Omega$ & $C_{\mathrm{Z}}=4.7 \mathrm{nF}$ \\
$C_{\mathrm{O}}=2200 \mu \mathrm{F}$ & $D_{3 \mathrm{VF}}=1.4 \mathrm{~V}$ & $C_{\mathrm{P}}=0 \mathrm{pF}$ \\
$R_{\mathrm{L}}=10 \Omega$ & $R_{1}=20 \mathrm{k} \Omega$ & $D_{\mathrm{EA} \mathrm{VF}}=2.5 \mathrm{~V}$ \\
$V_{\mathrm{O} \text { nom }}=30 \mathrm{~V}$ & $R_{2}=10 \mathrm{k} \Omega$ & $I C_{1 \mathrm{VF}}=1.1 \mathrm{~V}$ \\
& $Z D_{1 \mathrm{VZ}}=1 \mathrm{~V}$ & $I C_{1 \text { CTR }}=2$ \\
& & $I C_{1 \mathrm{VCE} \text { (sat) }}=0.5 \mathrm{~V}$
\end{tabular}

\begin{tabular}{lll}
\hline Parameter $x_{\mathrm{i}}$ & Lower bound & Upper bound \\
\hline$V_{\mathrm{DC}}$ & $V_{\mathrm{DC} \text { min }}=100 \mathrm{~V}$ & $V_{\mathrm{DC} \max }=300 \mathrm{~V}$ \\
$L_{\mathrm{M}}$ & $L_{\mathrm{M} \text { min }}=330 \mu \mathrm{H}$ & $L_{\mathrm{M} \max }=470 \mu \mathrm{H}$ \\
$F_{\mathrm{S}}$ & $F_{\mathrm{S} \min }=95 \mathrm{kHz}$ & $F_{\mathrm{S} \max }=105 \mathrm{kHz}$ \\
$D_{1 \mathrm{VF}}$ & $D_{1 \mathrm{VF} \text { min }}=0.6 \mathrm{~V}$ & $D_{1 \mathrm{VF} \text { max }}=1.2 \mathrm{~V}$ \\
$R_{\text {Sense }}$ & $R_{\text {Sense min }}=0.1425 \Omega$ & $R_{\text {Sense max }}=0.1575 \Omega$ \\
\hline
\end{tabular}

Table IV. SQP Parameters for the closed-loop flyback converter of Figure 4

\begin{tabular}{|c|c|c|c|c|c|c|c|}
\hline$k$ & 0 & 1 & 2 & 3 & 4 & 5 & \\
\hline$V_{\mathrm{DC}}^{(k)}$ in $\mathrm{V}$ & 202.0 & 202.0 & 201.999 & 201.986 & 100.0 & 100.0 & \\
\hline$L_{\mathrm{M}}^{(k)}$ in $\mu \mathrm{H}$ & 380.0 & 330.0 & 330.0 & 330.0 & 330.0 & 330.0 & \\
\hline$F_{\mathrm{S}}^{(k)}$ in $\mathrm{kHz}$ & 100.0 & 100.0 & 100.0 & 100.0 & 99.8984 & 95.0 & \\
\hline$D_{1 \mathrm{VF}}^{(k)}$ in $\mathrm{V}$ & 0.7 & 0.7 & 1.2 & 1.2 & 1.2 & 1.2 & $\begin{array}{l}\text { Table V. } \\
\text {. }\end{array}$ \\
\hline$R_{\text {Sense }}^{(k)}$ in $\Omega$ & 0.14 & 0.14 & 0.1416 & 0.1575 & 0.1575 & 0.1575 & $\begin{array}{l}\text { SQP Method on } \\
\text { closed-loop flyback }\end{array}$ \\
\hline$I_{\mathrm{LM} \text { max }}^{(k)}$ in $\mathrm{A}$ & -2.215 & -2.368 & -2.387 & -2.38702 & -2.539 & -2.583 & converter of Figure 4 \\
\hline
\end{tabular}

Component values and parameters for the closed-loop flyback converter of Figure 4 
COMPEL results are obtained by applying the AFSA to the Circuit-Model of the closed-loop flyback 38,5 converter. The simulation time $t_{\text {Sim }}$ was set to $30 \mathrm{~ms}$. The results from the SQP method are shown in Figure 7 as horizontal lines, these are marked with $\left|I_{\mathrm{LM} \max }^{(k)}\right|$. The peak currents of the transient simulations exactly matches the SQP results, having the same colors.

\subsection{Performance comparison}

To compare the performance of the SQP method, an MC analysis and evolution strategy $(1+1)$ ES (Beyer, 2001) has been implemented in Maple as well. The implemented MC analysis uses the continuous uniform distribution over the parameter ranges. All methods use the same objective function (15). Table VI summarizes the iterations for the methods and results. The $(1+1)$ ES method and the SQP methods provide the same result; however, the number of iterations for convergence varies greatly. The MC method could not find the exact worst-case value even at higher iterations runs $k$. The total CPU time of the SQP method is significantly shorter than that of the other two (e.g. $\approx 1 / 9,1 / 95,1 / 7$ ), illustrating the advantage of the AFSA.

\subsection{A more complex example case}

The example of the worst-case tolerance analysis of a flyback converter in CCM was shown. The example can be extended by e.g. the discontinuous conduction mode (DCM), which arises when the inductor current is zero. Here, the analytic forward solver approach provides the additional needed state for DCM. The initial conditions of the state variables can be expressed as it was done in the CCM example. Both models of the flyback converter can also be combined to one model which also supports switching between CCM and DCM. The CCM model becomes invalid when $I_{\mathrm{LM} 10}$ is negative.

Figure 7.

Comparison SQP results and transient simulation

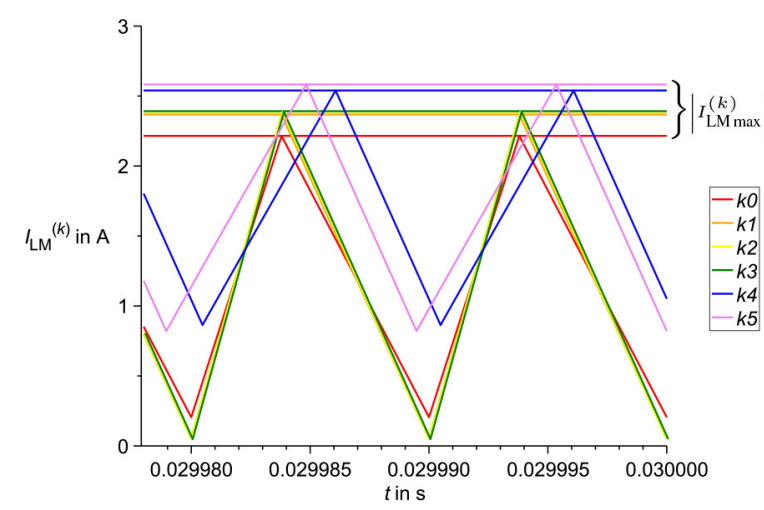

Table VI.

Performance comparison of different solutions methods

\begin{tabular}{lcccl}
\hline Method & MC & MC & $(1+1) \mathrm{ES}$ & SQP \\
\hline$\left|\hat{I}_{\text {LM }}\right|$ in A & 2.532 & 2.535 & 2.583 & 2.583 \\
$\# k$ iterations & 1000 & 10000 & 712 & 6 \\
CPU time in $s$ & 20.67 & 211.6 & 14.88 & 2.21 \\
\hline
\end{tabular}




\section{Conclusion}

The SQP method in combination with the AFSA shows that the worst-case limit converges in a few steps even if the worst-case limit is not on the boundary of the parameters. Based on the AFSA, it is possible to reduce a flyback converter in steady-state from a closed-loop to an open-loop system. These results are well in line with the transient simulation results obtained by applying AFSA to the open loop flyback converter. With respect to accuracy, the SQP method shows similar performance to $(1+1)$ ES strategy. Superior performance in terms of total CPU time was shown.

\section{References}

Analog Devices (2019), "LTspice”, available at: www.analog.com/en/design-center/design-tools-andcalculators/ltspice-simulator.html

Basso, C.P. (2012), Designing Control Loops for Linear and Switching Power Supplies: A Tutorial Guide, Artech House, Boston.

Beyer, D.H.-G. (2001), The Theory of Evolution Strategies, Natural Computing Series, 1st ed., SpringerVerlag Berlin Heidelberg.

Chiariello, A.G., Formisano, A., Martone, R. and Pizzo, F. (2015), "Gradient-based worst case search algorithm for robust optimization”, IEEE Transactions on Magnetics, Vol. 51 No. 3, pp. 1-4.

Cliquennois, S. and Trochut, S. (2007), "A new architecture simulator for integrated switched-mode power supplies circuits based on symbolic calculus tools", in Power Electronics Specialists Conference, 2007. PESC 2007. IEEE, pp. 224-230.

Fletcher, R. (2000), Practical Methods of Optimization, 2nd ed., John Wiley and Sons, Inc., England.

Fontana, G., Grasso, F., Luchetta, A., Manetti, S., Piccirilli, M.C. and Reatti, A. (2015), "A new simulation program for analog circuits using symbolic analysis techniques", International Conference on Synthesis, Modeling, Analysis and Simulation Methods and Applications to Circuit Design (SMACD), 2015, pp. 1-4.

Gielen, G.G.E., Walscharts, H.C.C. and Sansen, W. (1989), "Isaac: a symbolic simulator for analog integrated circuits”, IEEE Journal of Solid-State Circuits, Vol. 24 No. 6, pp. 1587-1597.

Khaligh, A., Rahimi, A.M., Khaligh, M. and Emadi, A. (2006), "Sensitivity analyses of pulse adjustment control technique of a buck-boost converter operating in discontinuous conduction mode and driving constant power loads", in 2006 IEEE Vehicle Power and Propulsion Conference, pp. 1-5.

Li, D. and Tymerski, R. (2000), "Comparison of simulation algorithms for accelerated determination of periodic steady state of switched networks", IEEE Transactions on Industrial Electronics, Vol. 47 No. 6, pp. 1278-1285.

Lian, K.L. (2012), "Derivation of a small-signal harmonic model for closed-loop power converters based on the state-variable sensitivity method", IEEE Transactions on Circuits and Systems I: Regular Papers, Vol. 59 No. 4, pp. 833-845.

Liberatore, A., Luchetta, A., Manetti, S. and Piccirilli, M.C. (1995), "A new symbolic program package for the interactive design of analog circuits", IEEE International Symposium on Circuits and Systems, 1995. ISCAS '95, 1995, Vol. 3, pp. 2209-2212.

Maplesoft (2019), "Maple 2017", available at: www.maplesoft.com

Moskovko, A. and Vityaz, O. (2018), "Transient analysis of electronic circuits using periodic steadystate analysis technique", in 2018 IEEE 38th International Conference on Electronics and Nanotechnology (ELNANO), pp. 499-503.

Nagel, L.W. (1975), "SPICE2: a computer program to simulate semiconductor circuits, PhD thesis, EECS department", University of California, Berkeley, available at: www.eecs.berkeley.edu/Pubs/ TechRpts/1975/9602.html 
COMPEL 38,5

Orcad PSpice (2019), “Orcad PSpice”, available at: www.orcad.com/products/orcad-pspice-designer/ overview

Ridley, D.R.B. (2011), Power Supply Design Volume 1: Control, Ridley Designs, Monpazier, France.

Setiawan, E., Hirata, T. and Hodaka, I. (2017), "Steady state symbolic analysis of buck converter using fourier series", in 2017 2nd International Conference on Frontiers of Sensors Technologies (ICFST), pp. 299-303.

Thomassian, J.-C. (2007), "Symbolic techniques: a new tool for teaching circuits and electronics", in Frontiers In Education Conference - Global Engineering: Knowledge Without Borders, Opportunities Without Passports, 2007. FIE '07. 37th Annual, pp. S2C-1-S2C-5.

Wong, B.K.H., Chung, H.S.H. and Lee, S.T.S. (2000), "Computation of the cycle state-variable sensitivity matrix of pwm dc/dc converters and its applications", IEEE Transactions on Circuits and Systems I: Fundamental Theory and Applications, Vol. 47 No. 10, pp. 1542-1548.

\section{Corresponding author}

Mario Schenk can be contacted at: mario.schenk@student.tugraz.at

For instructions on how to order reprints of this article, please visit our website: 